\title{
Entrepreneurship education today for students' unknown futures
}

\author{
Bethany Hardie ${ }^{1}$, Camilla Highfield ${ }^{2}$ and Kerry Lee ${ }^{3}$ \\ ${ }^{1}$ The University of Auckland, New Zealand (ORCID: 0000-0001-5493-5096) \\ ${ }^{2}$ The University of Auckland, New Zealand (ORCID: 0000-0001-8896-1989) \\ ${ }^{3}$ The University of Auckland, New Zealand (ORCID: 0000-0002-6410-3688)
}

\begin{abstract}
Society requires education to prepare students with the tools and ability to navigate and find success for unknown futures. Entrepreneurship education has the potential to deliver the relevant curriculum and competencies to support young people to develop resilience, independence, innovation and ability to recognise opportunities to live productive and rewarding lives in this new post COVID-19 environment. Entrepreneurship has been encouraged by government initiatives to address rapidly evolving challenges due to economic disruption. The purpose of this study was to conduct a literature review of entrepreneurship education pedagogy in order to understand the growing evidence of the effectiveness of programmes that support students to act on opportunities that address social, economic, and environmental issues that have arisen in their communities. An analysis of 45 studies across nine countries suggests that although these types of learning opportunities are written into curricula, students rarely experience this type of learning in their schooling. Sustained interest in entrepreneurship through effective methods such as assistance from external trainers and value creation throughout schooling develops students' intent for continued study of entrepreneurship at university. Teachers need opportunities to build confidence, knowledge and capacity to develop effective entrepreneurship education learning experiences that are relevant to today's students' future life challenges.
\end{abstract}

Keywords: Entrepreneurship; Enterprise; COVID-19; Education; School

Article History: Submitted 1 July 2020; Revised 1 September 2020; Published online 3 September 2020

\section{Introduction}

Our global 'new normal,' we are told, is a state of disruption with an economic recession forecast to match if not surpass the Great Depression (Baker et al., 2020, para. 21). As of May 26, 2020, over 5.6 million people have contracted the COVID-19 virus, over 340,000 people have died, and 213 countries and territories have been affected (Worldometer, 2020). 'Lockdowns' are our new vernacular and have confined people to their homes. Parents have balanced online schooling with online work as businesses and schools have had to temporarily close to stop the virus spreading. Lockdowns imposed to contain the virus shattered the economy and millions of people have been forced into unexpected unemployment. Businesses have been closed beyond their capacity to reopen, global trade chains broken, manufacturing stopped and started, borders closed to travel

Address of Corresponding Author

Kerry Lee, PhD, Technology Education in the School of Curriculum and Pedagogy, Faculty of Education and Social Work, The University of Auckland, New Zealand.

$\triangle$ k.lee@auckland.ac.nz

How to cite: Hardie, B., Highfield, C., \& Lee, K. (2020). Entrepreneurship education today for students' unknown futures. Journal of Pedagogical Research, 4(3), 401-417. 
devastating the tourism and hospitality industry, and industries stigmatised as potential virus spreaders have been shunned (Akkermans et al., 2020; Bhattacharjee \& Jahanshah, 2020; Gössling et al., 2020; Haeffele et al., 2020). The continued spiralling down of the global economy means that large scale redundancies leads to more unemployment as constrained household budgets tighten purse strings.

New Zealand has been fortunate to have contained the virus and while still refining the border control as citizens return to keep the virus out of the country, the government is focused on propping up the economy and an entrepreneurial approach to recovery is recognised as vital (Robertson, 2020). 'An Early Mover's Initiative of Nations,' involves leaders from nine countries that have contained COVID-19, and together have discussed the best way to reopen the economy (Wade, 2020). New Zealand is part of this group along with Australia, Austria, Czech Republic, Demark, Greece, Israel, Norway, and Singapore. Internationally, small and medium enterprises (SME) have been hit hard by the economy shut down (Akkermans et al., 2020) yet are seen as key to a recovery plan (Dai et al., 2020; Fernandes, 2020; McKee \& Stuckler, 2020; Robertson, 2020). Up until COVID-19, SME have added almost three quarters of the total jobs in the United States each year (Harrington \& Maysami, 2015) and are recognised as pivotal to creating employment and reducing the gap between rich and poor (Nseobot et al., 2020). The talents and passions of students can be realised through the creation of SME businesses (Kirkley, 2017). How is public education building entrepreneurial intent in learners so that they envision using their talents and passion as SME e-commerce owners that can create much needed employment and equity in revenue in a battered and recovering economy?

Students have endured isolation from school which provides essential social interaction for many young people. Formal assessments have been cancelled or changed, family stress has increased, while routines have been restricted and modified (Bryant et al., 2020; OECD, 2020; UNESCO, 2020; Van Lancker \& Parolin, 2020). Schools provide many students with lunches, help regulate behaviour, and keep a connection to mitigate family issues (OECD, 2020; UNESCO, 2020). In addition to providing more support to vulnerable groups, the purpose of education needs to be re-evaluated to better support student wellbeing and resilience (OECD, 2020; UNESCO, 2020). Public education needs to empower learners with competencies that take time to develop (Lackéus, 2015; Rieckmann, 2020) and magnetise student engagement so that students develop resilience behaviours and remain connected (UNESCO, 2020). It is of concern that so many students have been reported to lack intrinsic competencies and motivation to construct their own learning while at home (Bryant et al., 2020; OECD, 2020; Reimers \& Schleicher, 2020; UNESCO, 2020; Van Lancker \& Parolin, 2020; Zhou et al., 2020).

Public education providers need to lean into a new normal with a localised and contextualised curriculum that encourages creativity and innovation, supports wellbeing and resilience, (OECD, 2020) develops competencies (Rieckmann, 2020) and promotes student engagement and selfmotivation (UNESCO, 2020). In this paper we provide evidence that entrepreneurship education provides the opportunity for students to gain meaning from their learning and develop the types of competencies that will support them throughout their lives. This literature review of entrepreneurship education pedagogy provides growing evidence of the effectiveness of programmes that support students to act on opportunities that address social, economic, and environmental issues that have arisen in their communities.

\subsection{Theoretical Framework}

Entrepreneurship education is an approach to learning that has been adopted across Asia, in China, Indonesia, (Wu \& Wu, 2017), Singapore (Ho et al., 2018) and Malaysia (Din et al., 2016), in most of the European Union countries, and in the United States, with the European Union countries taking a more practical approach (Ierapetritis, 2017). Global interest grew on the topic of entrepreneurship in the early 1980s as the economy's focus turned to small and medium-sized (SME) businesses as a solution to unemployment (Jones \& Iredale, 2014; Pepin, 2018). In Finland 
(Komulainen et al., 2009) and Sweden (Fejes et al., 2019) entrepreneurship education is written into the curriculum for all year levels and across all subjects. In the United States, 'entrepreneurship education' is the terminology used; while 'enterprise education' is used in the United Kingdom (Lackéus \& Middleton, 2015); and in New Zealand's curriculum students are to explore what it is to be 'enterprising' (Ministry of Education, 2011). As an approach to education, enterprise and entrepreneurship students are supported to be innovative and creative (Gibb \& Ramsey, 2011), encouraged to use their initiative and be adaptable (Dahlstedt \& Hertzberg, 2012; Smith \& Price, 2011).

\subsubsection{A narrow or broad view of entrepreneurship education}

There are two perspectives on entrepreneurship education. A narrow view of entrepreneurial education is thought to confine entrepreneurship to a business subject that provides an opportunity to gain knowledge and understanding about marketing, finance, human resource development, strategy and acquiring capital (Lackéus, 2015; Osiri et al., 2015). Taking a broader view of entrepreneurial competencies requires learning through ventures or designs that respond to opportunities in a wider range of subject areas and problem solving opportunities throughout the surrounding community and can appeal to students who are interested in ventures that serve a broader purpose in society (Kirkley, 2017; Osiri et al., 2015). While some argue that the narrow and broad views be kept separate (Jones \& Iredale, 2014) and there is preference for the broader view (Davidsen, 2015; Lackéus, 2015), these views can co-exist (Fejes et al., 2019) when students learn about, for, and through entrepreneurship (Hannon, 2005).

\subsubsection{Learning about, for or through entrepreneurship education}

In a public education or schooling context, entrepreneurship education experiences can be designed to teach students about, for, or through entrepreneurship. Learning about entrepreneurship has been described as learning the 'what' and 'how' of entrepreneurship (Nabi et al., 2018), by presentations of the associated theories and knowledge (Lackéus, 2015). Learning for entrepreneurship is preparing students to start a business (Caird, 1990; Moberg, 2014) with technical, practical and teacher-guided instruction (Elahi, 2019; Sirelkhatim \& Gangi, 2015). Learning through or in entrepreneurship is to experience real life ventures (Piperopoulos \& Dimov, 2015) to develop skills and competencies in entrepreneurship (Caird, 1990; Lackéus, 2015; Moberg, 2014; Sirelkhatim \& Gangi, 2015). Age has been related to approach (Figure 1) by Dahlstedt and Hertzberg (2012) and (Lackéus, 2015) with learning through and for suited to primary and secondary school students while about suited to university level . However, there is growing consensus that learning through entrepreneurship education is more effective even for tertiary level (Kleiman, 2015; O'Leary, 2012; Scharmer et al., 2020). Learning for entrepreneurship has been found to be more effective in fostering entrepreneur identity at lower secondary school level but less engaging (Moberg, 2014), and while still worthwhile, should be presented as a variety of ways learners could act, considering complex conditions, rather than taught instrumentally (Rieckmann, 2020).

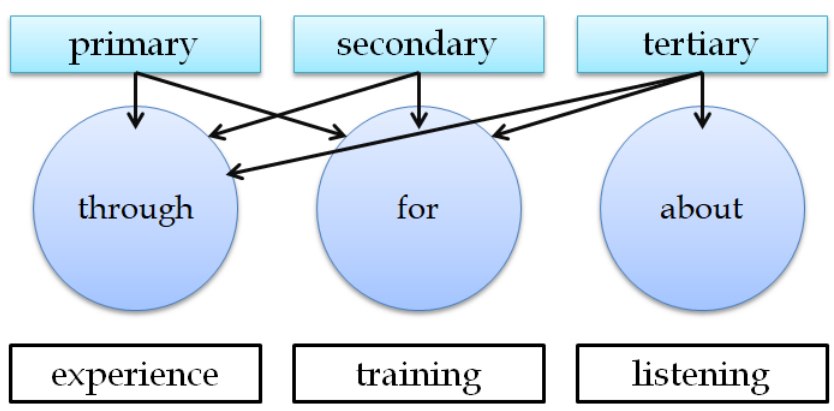

Figure 1. Entrepreneurship education approaches in relation to education level. (Author based on work by Dahlstedt \& Hertzberg, 2012; Kleiman, 2015; Lackéus, 2015; O'Leary, 2012; Rieckmann, 


\section{Method}

A literature review was conducted using the systematic review method outlined by Tranfield et al. (2003). This method was also used by $\mathrm{Wu}$ and $\mathrm{Wu}$ (2017) in their review of entrepreneurship education across Asia-Pacific countries. The systematic review method allows the researcher to gather literature from different methods and contexts in order to elicit themes and areas that require further research (Tranfield et al., 2003). There are three stages according to Tranfield et al. (2003) to carrying out a systematic review; 'planning the review', 'conducting a review', and 'reporting and dissemination (p.214).'

\subsection{Research Design}

The review was planned in the first stage to sample perspectives from a range of countries that provided entrepreneurship education studies that had measured the effectiveness of the programmes in terms of outcomes for students. As Tranfield et al. (2003) state, a procedure must be planned in the first stage for selecting studies in the second stage to be synthesised and included in the review. In the third stage the researcher produces a report and distributes the findings. The final inclusion criteria are set out in Table 1.

In the second stage the keywords used to identify the literature included; 'entrepreneurship education', 'enterprise education,' and 'effectiveness' or 'school' or 'primary' or 'secondary' or 'pedagogy.' Once a range of countries had been determined following initial searches of those terms, searches would also include the name of the country. The articles were recorded and summarised in a table to include reference and country, design assessment measures, age range and group, focus of the study, number of participants, and outcomes/findings.

It was found that the initial inclusive criteria 1, which was for an even distribution of five studies per country needed to be adjusted to improve the assessment of relevance to the purpose of the study. Searches that related to the study for Finland found seven studies whereas New Zealand and Netherlands each had four. Three studies from Finland were excluded to improve the distribution of studies across countries; one had a very small sample size, one was an earlier study of an author already included and one was more focused on politics and considered less pertinent to the purpose of this study. University studies and longitudinal studies on the schooling of entrepreneurs were also found to be relevant so criteria 1 was amended and criteria 6 was introduced. Criteria 6 however, excluded the United States which had 4 out of 5 studies from university level and Germany as all three studies found were at university level. As Tranfield et al. (2003) explains, assessment of the relevance to the study informs the researcher during the selection process.

Table 1

The Procedure for Selecting Studies for the Review Based on Inclusion and Exclusion

\begin{tabular}{|c|c|}
\hline Inclusion & \\
\hline $\begin{array}{l}\text { Studies related to the topic of the } \\
\text { effectiveness of entrepreneurship or } \\
\text { enterprise education in public education }\end{array}$ & n \\
\hline $\begin{array}{l}\text { accumulated evidence for } \\
\text { acto and } 2020\end{array}$ & $\begin{array}{l}\text { a new contribution to the } \\
\text { study }\end{array}$ \\
\hline $\begin{array}{l}\text { At least two studies relating to } \\
\text { entrepreneurship education in primary and } \\
\text { secondary schools for one country }\end{array}$ & $\begin{array}{l}>\text { Countries that resulted in less than two } \\
\text { studies from primary and secondary schools } \\
>\text { Literature that had not carried out a study }\end{array}$ \\
\hline
\end{tabular}


Identification of research, selection of studies, quality assessment, data extraction, monitoring process and data synthesis was carried out in stage two of the systematic review. The criteria for the procedure resulted in forty five studies gathered across nine countries over a period of two weeks that related to the effectiveness of primary and secondary entrepreneurship education within the period 2010 to 2020.

During the third stage of the systematic review, an interpretative approach was used to carry out a thematic analysis and synthesise findings across the 45 studies. The third stage was completed through producing the report and dissemination of the recommendations.

\subsection{Participants}

The age range, group information and number of participants for each study were recorded in a table during data extraction (Table 2).

\subsection{Data Collection Instruments}

The research consisted of using Google Scholar and The University of Auckland search engines to find journal articles that had conducted studies into effectiveness of entrepreneurship education in primary, secondary and tertiary levels (Figure 2). These studies aimed to provide empirical evidence on the outcomes of entrepreneurship education from the viewpoint of students, teachers, lecturers, principals, or working adults. The review sought to understand the effectiveness of programmes in developing students' attitudes, skills, and competencies towards entrepreneurship.

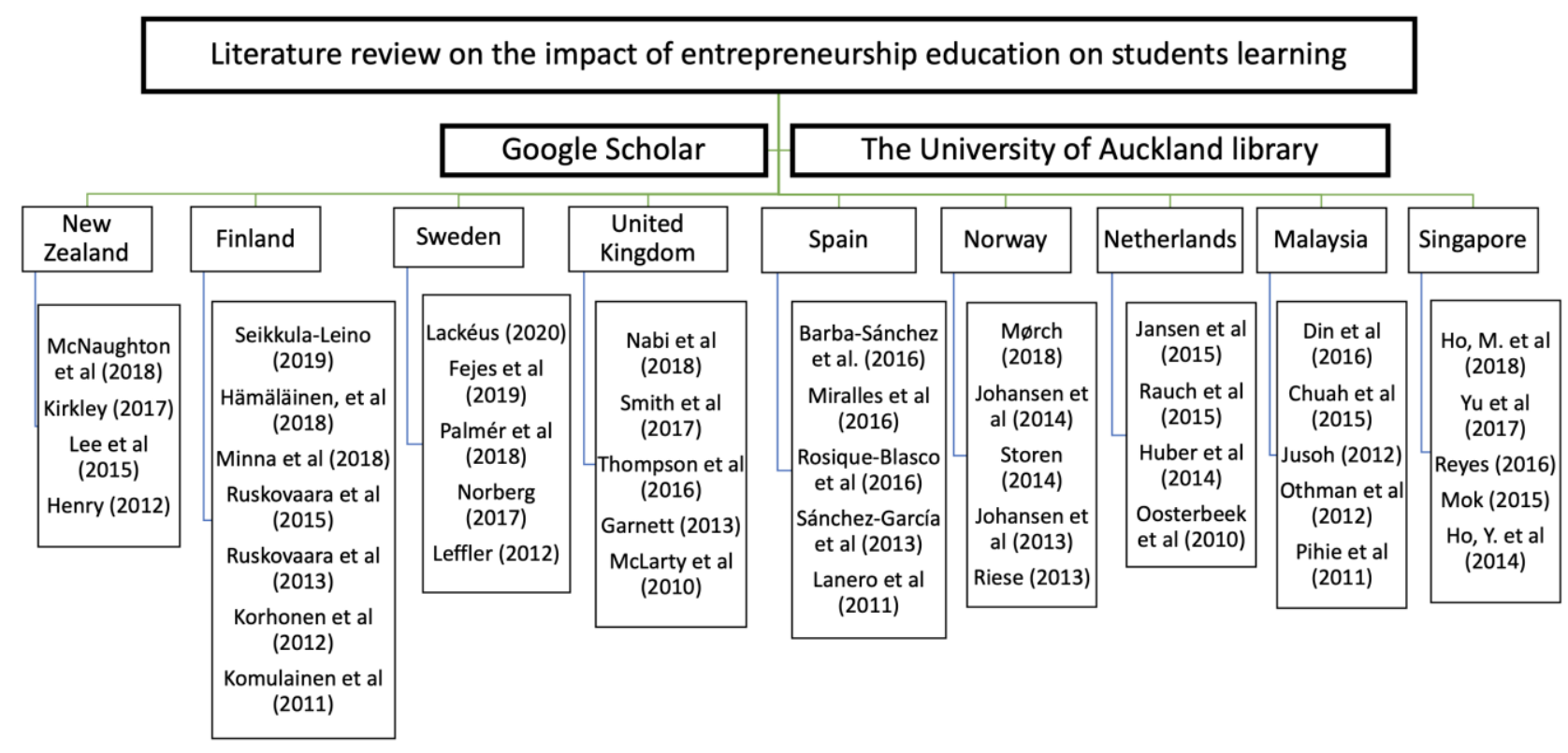

Figure 2. Distribution of literature ordered according to country and year of publication

\section{Results}

The findings are presented according to the four themes that arose in synthesising the studies.

\subsection{Benefits for Students from Entrepreneurship Education}

Within the 45 articles on entrepreneurship education, 18 studies investigated the benefits to students. An overview of these studies are listed in Table 2 with descriptions of the benefits found and summarised in Figure 3. 
Table 2

Within a Sample of 45 Articles Collected to Understand the Effectiveness of Entrepreneurship Pedagogy in Nine Countries, 18 Studies Focused on the Benefits of Entrepreneurship Education for Students

\begin{tabular}{|c|c|c|c|c|c|}
\hline $\begin{array}{l}\text { References } \\
\& \\
\text { country }\end{array}$ & $\begin{array}{l}\text { Design } \\
\text { Assessment } \\
\text { measures }\end{array}$ & $\begin{array}{l}\text { Age Range } \\
\text { \& Group } \\
\text { information }\end{array}$ & Focus of study & $\begin{array}{l}\text { Number of } \\
\text { participants } \\
(n)\end{array}$ & $\begin{array}{l}\text { Outcomes/ } \\
\text { Findings }\end{array}$ \\
\hline $\begin{array}{l}\text { Lackéus. } \\
(2020) \\
\text { Sweden }\end{array}$ & Survey, SSI & $\begin{array}{l}8 \text { to } 15 \text { year } \\
\text { olds }\end{array}$ & $\begin{array}{l}\text { Comparison of EE } \\
\text { methods }\end{array}$ & $\begin{array}{c}\mathrm{Q} \\
\mathrm{n}=1048 \\
\mathrm{SSI} \\
\mathrm{n}=291\end{array}$ & 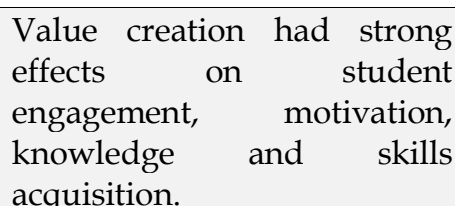 \\
\hline $\begin{array}{l}\text { Ho et al. } \\
\quad(2018) \\
\text { Singapore }\end{array}$ & $\begin{array}{l}\text { Survey } \\
\text { Quasi-expe- } \\
\text { rimental }\end{array}$ & $\begin{array}{l}13 \text { to } 16 \text { year } \\
\text { olds }\end{array}$ & $\begin{array}{l}\text { Impact of } \\
\text { entrepreneurship } \\
\text { training }\end{array}$ & $n=328$ & $\begin{array}{l}\text { Higher entrepreneurial skill } \\
\text { set and mindset efficacies, } \\
\text { and improved ability to scan } \\
\text { and search for, and evaluate } \\
\text { and judge entrepreneurial } \\
\text { opportunities. }\end{array}$ \\
\hline $\begin{array}{l}\text { Nabi et al. } \\
\text { (2018) } \\
\text { United } \\
\text { Kingdom }\end{array}$ & $\begin{array}{l}\text { Survey, } \\
\text { SSI } \\
\text { longitudinal }\end{array}$ & $\begin{array}{l}\text { First year } \\
\text { university } \\
\text { students }\end{array}$ & $\begin{array}{l}\text { Role of EE in } \\
\text { fostering } \\
\text { entrepreneurship } \\
\text { intentions }\end{array}$ & $\begin{array}{c}\mathrm{Q} \\
\mathrm{n}=150 \\
\mathrm{SSI} \\
\mathrm{n}=49\end{array}$ & $\begin{array}{l}\text { Demonstrated higher } \\
\text { entrepreneurial learning, } \\
\text { inspiration and intentions as } \\
\text { long as the learning } \\
\text { experiences were positive }\end{array}$ \\
\hline $\begin{array}{l}\text { Palmer et al. } \\
\text { (2018) } \\
\text { Sweden }\end{array}$ & $\begin{array}{l}\text { Design } \\
\text { research }\end{array}$ & $\begin{array}{l}\text { Teachers of } \\
6 \text { to } 12 \text { year } \\
\text { olds }\end{array}$ & $\begin{array}{l}\text { EE integrated into } \\
\text { maths }\end{array}$ & $\mathrm{n}=30$ & $\begin{array}{l}\text { Entrepreneurship } \\
\text { competencies were of positive } \\
\text { value in maths lessons. } \\
\text { (Creativity, resilience, } \\
\text { courage, initiative, } \\
\text { collaboration, } \\
\text { responsibility) }\end{array}$ \\
\hline $\begin{array}{l}\text { Kirkley } \\
(2017) \\
\text { New } \\
\text { Zealand }\end{array}$ & FG, SSI & $\begin{array}{r}\text { Secondary } \\
\text { Schools }\end{array}$ & $\begin{array}{l}\text { Effectiveness of } \\
\text { EE }\end{array}$ & $n=6123$ & $\begin{array}{l}\text { Reduced direct teaching } \\
\text { workload. Students' attitudes } \\
\text { improved with more } \\
\text { engagement in school }\end{array}$ \\
\hline $\begin{array}{l}\text { Barba- } \\
\text { Sanchez et } \\
\text { al. (2016) } \\
\text { Spain }\end{array}$ & $\begin{array}{l}\text { Quasi- } \\
\text { experimental }\end{array}$ & $\begin{array}{l}\text { Primary } \\
\text { school } \\
\text { Year 3 - } 6\end{array}$ & $\begin{array}{l}\text { Effectiveness of } \\
\text { EE }\end{array}$ & $n=49$ & $\begin{array}{l}\text { Entrepreneurship intent rose } \\
\text { from } 0 \% \text { to } 56 \% \text { after two } \\
\text { years of EE }\end{array}$ \\
\hline $\begin{array}{l}\text { Din et al. } \\
\text { (2016) } \\
\text { Malaysia }\end{array}$ & Survey & $\begin{array}{l}\text { University } \\
\text { students }\end{array}$ & $\begin{array}{l}\text { Effectiveness of } \\
\text { EE }\end{array}$ & & $\begin{array}{l}\text { Improves entrepreneurship } \\
\text { skills, self efficacy, business } \\
\text { plans and risk thinking }\end{array}$ \\
\hline $\begin{array}{l}\text { Miralles et } \\
\text { al. (2016) } \\
\text { Spain }\end{array}$ & Survey & $\begin{array}{l}\text { Average age } \\
\text { of } 35 \text { years }\end{array}$ & $\begin{array}{l}\text { Entrepreneurial } \\
\text { intent in adults }\end{array}$ & $n=431$ & $\begin{array}{l}\text { Knowledge } \\
\text { entrepreneurship increased } \\
\text { entrepreneurial intent }\end{array}$ \\
\hline $\begin{array}{l}\text { Thompson } \\
\text { et al. (2016) } \\
\text { United } \\
\text { Kingdom }\end{array}$ & $\begin{array}{l}\text { GEM data } \\
\text { ph calls }\end{array}$ & $18-45 y r s$ & $\begin{array}{l}\text { Influence of EE on } \\
\text { career }\end{array}$ & $n=16343$ & $\begin{array}{l}\text { Students from compulsory } \\
\text { courses in EE were two and } \\
\text { half times more likely to take } \\
\text { part in university and } \\
\text { government ventures. } \\
\text { Those in voluntary EE were } \\
\text { six times more likely to } \\
\text { continue to higher levels. }\end{array}$ \\
\hline $\begin{array}{l}\text { Rauch et al. } \\
\quad(2015) \\
\text { Netherlands }\end{array}$ & $\begin{array}{l}\text { Pre- and } \\
\text { post- test }\end{array}$ & $\begin{array}{l}\text { University } \\
\text { students }\end{array}$ & $\begin{array}{l}\text { Effectiveness } \\
\text { EE }\end{array}$ & $n=96$ & $\begin{array}{l}\text { Increased attitudes, perceived } \\
\text { behavioural control and } \\
\text { entrepreneurship intentions }\end{array}$ \\
\hline
\end{tabular}


Table 2 continued

\begin{tabular}{|c|c|c|c|c|c|}
\hline $\begin{array}{l}\text { References } \\
\& \\
\text { country }\end{array}$ & $\begin{array}{l}\text { Design } \\
\text { Assessment } \\
\text { measures }\end{array}$ & $\begin{array}{l}\text { Age Range } \\
\text { \& Group } \\
\text { information }\end{array}$ & Focus of study & $\begin{array}{l}\text { Number of } \\
\text { participants } \\
(n)\end{array}$ & $\begin{array}{l}\text { Outcomes/ } \\
\text { Findings }\end{array}$ \\
\hline $\begin{array}{l}\text { Mok (2015) } \\
\text { Singapore }\end{array}$ & Survey & $\begin{array}{l}\text { University } \\
\text { teaching } \\
\text { staff }\end{array}$ & $\begin{array}{l}\text { Effectiveness of } \\
\text { EE }\end{array}$ & $\mathrm{n}=208$ & $\begin{array}{l}\text { EE enhances quality of } \\
\text { graduates }\end{array}$ \\
\hline $\begin{array}{l}\text { Ho et al. } \\
\text { (2014) }\end{array}$ & Survey & University & Impact of EE & $n=836$ & $\begin{array}{l}\text { Venture creation had a } \\
\text { significant positive } \\
\text { influence on } \\
\text { entrepreneurial } \\
\text { engagement. }\end{array}$ \\
\hline $\begin{array}{l}\text { Huber et al. } \\
\quad(2014) \\
\text { Netherlands }\end{array}$ & $\begin{array}{l}\text { Survey \& } \\
\text { observed }\end{array}$ & $\begin{array}{l}\text { Primary } \\
\text { school } \\
\text { Yr } 6\end{array}$ & $\begin{array}{l}\text { Effectiveness of } \\
\text { EE through } \\
\text { 'BizWorld' }\end{array}$ & $\begin{array}{c}\text { Treatment } \\
\mathrm{n}=1729 \\
\text { Control } \\
\mathrm{n}=684\end{array}$ & $\begin{array}{l}\text { Treatment group developed } \\
\text { more efficiency in } \\
\text { entrepreneurial non } \\
\text { cognitive skills for risk } \\
\text { taking, self-efficacy, } \\
\text { creativity, need for } \\
\text { achievement, persistence, a } \\
\text { 'can do' attitude, and } \\
\text { analysing. Improved teacher } \\
\text { motivation and knowledge } \\
\text { of EE }\end{array}$ \\
\hline $\begin{array}{l}\text { Støren } \\
(2014) \\
\text { Norway }\end{array}$ & Survey & $\begin{array}{l}\text { University } \\
\text { graduates }\end{array}$ & $\begin{array}{l}\text { Effectiveness of } \\
\text { EE }\end{array}$ & $\mathrm{n}=2827$ & $\begin{array}{l}\text { Increased entrepreneurial } \\
\text { skills } \\
\text { through=skills } \\
\text { for=increased ability to } \\
\text { start-up business }\end{array}$ \\
\hline $\begin{array}{l}\text { Garnett. } \\
\text { (2013) } \\
\text { United } \\
\text { Kingdom }\end{array}$ & $\begin{array}{l}\text { Action } \\
\text { research, } \\
\text { Survey, } \\
\text { Journals, } \\
\text { SSI }\end{array}$ & $\begin{array}{l}\text { Teachers a } \\
\text { students at } \\
3 \text { schools }\end{array}$ & $\begin{array}{l}\text { Effectiveness of } \\
\text { EE }\end{array}$ & $\begin{array}{l}\text { Teachers } \\
\mathrm{n}=3 \\
\text { Students } \\
\mathrm{n}=79\end{array}$ & $\begin{array}{l}\text { Students motivated by } \\
\text { creativity and ownership of } \\
\text { learning. Students liked } \\
\text { learning new skills. }\end{array}$ \\
\hline $\begin{array}{l}\text { Lanero et al. } \\
\text { (2011) } \\
\text { Spain }\end{array}$ & Survey & $\begin{array}{l}\text { University } \\
\text { students }\end{array}$ & Impact of EE & $\mathrm{n}=800$ & $\begin{array}{l}\text { Increased feasibility, intent, } \\
\text { and student involvement for } \\
\text { entrepreneurship }\end{array}$ \\
\hline $\begin{array}{l}\text { Pihie et al. } \\
\text { (2011) } \\
\text { Malaysia }\end{array}$ & Survey & $\begin{array}{l}\text { Secondary } \\
\text { school } \\
\text { students }\end{array}$ & $\begin{array}{l}\text { Entrepreneurship } \\
\text { intention }\end{array}$ & $\mathrm{n}=2574$ & 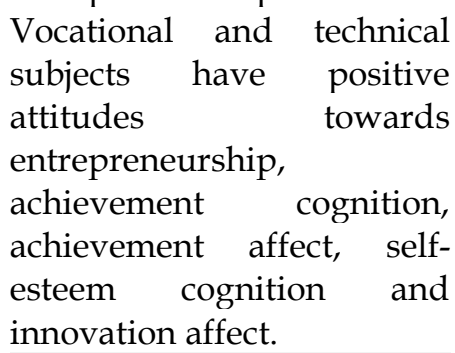 \\
\hline $\begin{array}{l}\text { McLarty et } \\
\text { al. (2010) } \\
\text { United } \\
\text { Kingdom }\end{array}$ & $\begin{array}{l}\text { Survey, FG } \\
\& \\
\text { observed }\end{array}$ & $\begin{array}{l}\text { Secondary } \\
\text { schools }\end{array}$ & $\begin{array}{l}\text { Perceived impact } \\
\text { of EE }\end{array}$ & $\begin{array}{c}\text { Schools } \\
n=408\end{array}$ & 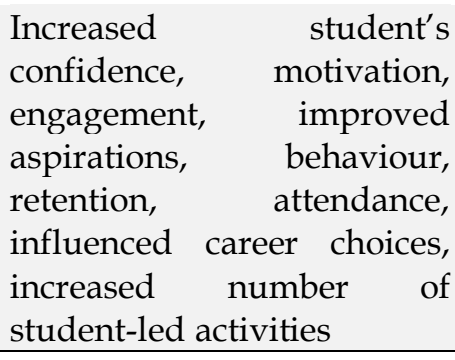 \\
\hline
\end{tabular}

Q: questionnaire/survey; SSI: semi-structured interview; FG: focus groups 


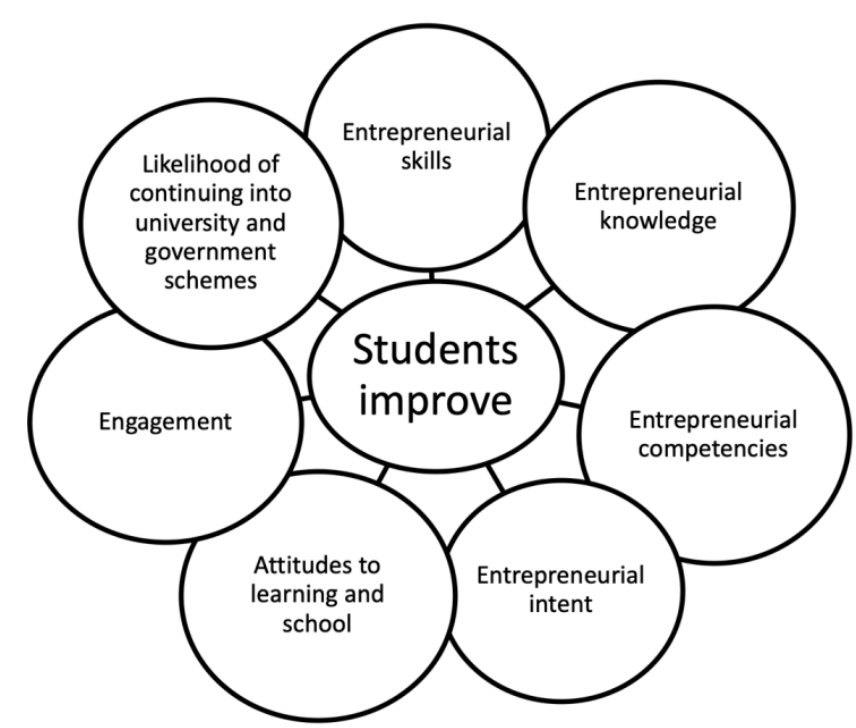

Figure 3. Categories from thematic analysis of the theme 'benefits of entrepreneurship education' found within 18 studies that focused on student outcomes

\subsection{Developing Entrepreneurial Intent}

The theory of planned behaviour (Ajzen, 1991) was often used to show how entrepreneurial intent could be developed (Figure 4).

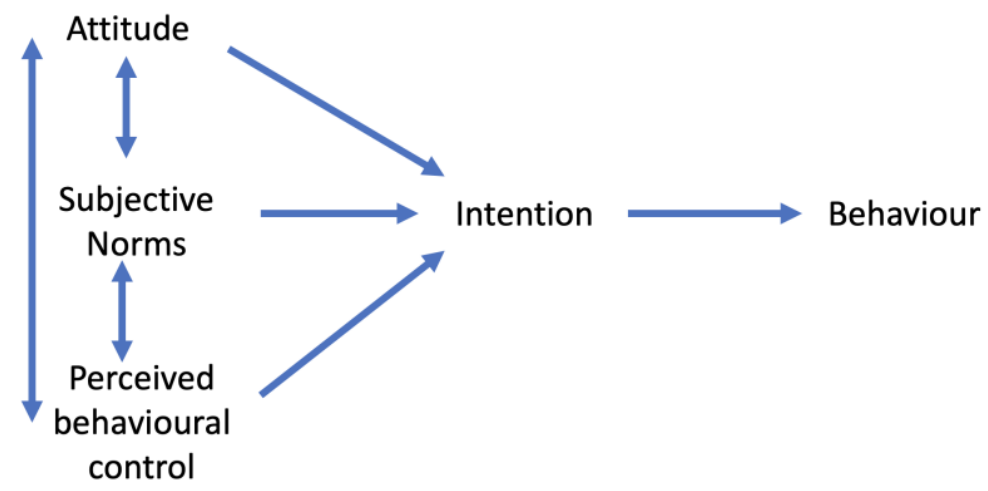

Figure 4. The theory of planned behaviour (Ajzen, 1991)

This theory was used in Spain by Lanero et al. (2011) in order to study 800 university students' intentions towards entrepreneurship and found intentions were related to perceived behavioural control and perceived feasibility of entrepreneurship. Miralles et al. (2016), also in Spain, used the theory to study entrepreneurship intent in 431 working adults and found that knowing about entrepreneurship and seeing it as an attractive career choice influenced intention. Chuah et al. (2015) used the theory in Malaysia to study entrepreneurial intent in 204 university students. Positive attitude, subjective norm, image, finances and perceived behavioural control were found to increase intent. Ho et al. (2018) used the theory and found positive levels of engagement in 836 secondary school students when using venture creation in Singapore.

Four studies recommended that intent be developed earlier in education in order to build and maintain entrepreneurial intent in learners (see Table 3 and Figure 5). 
Table 3

Studies with Findings and Recommendations to Develop Entrepreneurial Intent

\begin{tabular}{|c|c|c|c|c|c|}
\hline $\begin{array}{c}\text { References } \\
\& \\
\text { country }\end{array}$ & $\begin{array}{l}\text { Design } \\
\text { Assessment } \\
\text { measures }\end{array}$ & $\begin{array}{l}\text { Age Range } \\
\text { \& Group } \\
\text { information }\end{array}$ & Focus of study & $\begin{array}{l}\text { Number of } \\
\text { participants } \\
(n)\end{array}$ & $\begin{array}{l}\text { Outcomes/ } \\
\text { Findings }\end{array}$ \\
\hline $\begin{array}{l}\text { Nabi et al. } \\
\text { (2018) } \\
\text { United } \\
\text { Kingdom }\end{array}$ & $\begin{array}{l}\text { Survey \& } \\
\text { Interview }\end{array}$ & $\begin{array}{l}\text { First year } \\
\text { university } \\
\text { students 18- } \\
25 \text { year old }\end{array}$ & $\begin{array}{l}\text { The role of EE in } \\
\text { developing } \\
\text { intent }\end{array}$ & $\begin{array}{c}\text { Survey } \\
\mathrm{n}=150 \\
\text { Interview } \\
\mathrm{n}=49\end{array}$ & $\begin{array}{l}\text { An accumulation of positive } \\
\text { experiences for university } \\
\text { students lead to a strong } \\
\text { increase in entrepreneurial } \\
\text { intentions. }\end{array}$ \\
\hline $\begin{array}{l}\text { Din et al. } \\
(2016) \\
\text { Malaysia }\end{array}$ & Survey & $\begin{array}{l}\text { University } \\
\text { students }\end{array}$ & $\begin{array}{l}\text { Effectiveness of } \\
\text { EE }\end{array}$ & $\mathrm{n}=130$ & $\begin{array}{l}\text { Enhanced self-efficacy, risk } \\
\text { taking and business plan } \\
\text { skills } \\
\text { Recommended: allowing } \\
\text { primary school students to } \\
\text { explore their interests } \\
\text { through entrepreneurship } \\
\text { education, then start to } \\
\text { introduce the basic steps in } \\
\text { high school as preparation } \\
\text { for deeper knowledge in } \\
\text { university }\end{array}$ \\
\hline $\begin{array}{l}\text { Rosique-Bla } \\
\text { sco et al. } \\
\text { (2016) } \\
\text { Spain }\end{array}$ & Survey & $\begin{array}{l}\text { Secondary } \\
\text { school } \\
\text { students }\end{array}$ & $\begin{array}{l}\text { How skills \& } \\
\text { socio-cultural } \\
\text { factors affect } \\
\text { intent }\end{array}$ & $\mathrm{n}=1244$ & $\begin{array}{l}\text { Found: } \\
\text { creativity, proactivity, risk } \\
\text { taking, and role models } \\
\text { promote entrepreneurial } \\
\text { intent. }\end{array}$ \\
\hline $\begin{array}{l}\text { McLarty et } \\
\text { al. (2010) } \\
\text { United } \\
\text { Kingdom }\end{array}$ & $\begin{array}{l}\text { Survey \& } \\
\text { observed }\end{array}$ & $\begin{array}{l}8 \text { to } 10 \text { year } \\
\text { olds }\end{array}$ & $\begin{array}{l}\text { Effectiveness of } \\
\text { EE }\end{array}$ & $\mathrm{n}=130$ & $\begin{array}{l}\text { Recommended: students } \\
\text { become more involved in } \\
\text { businesses at a younger age } \\
\text { so that they are aware of } \\
\text { their capabilities and career } \\
\text { options as they make } \\
\text { vocational choices in } \\
\text { subjects at aged } 14 .\end{array}$ \\
\hline
\end{tabular}

EE: Entrepreneurship education

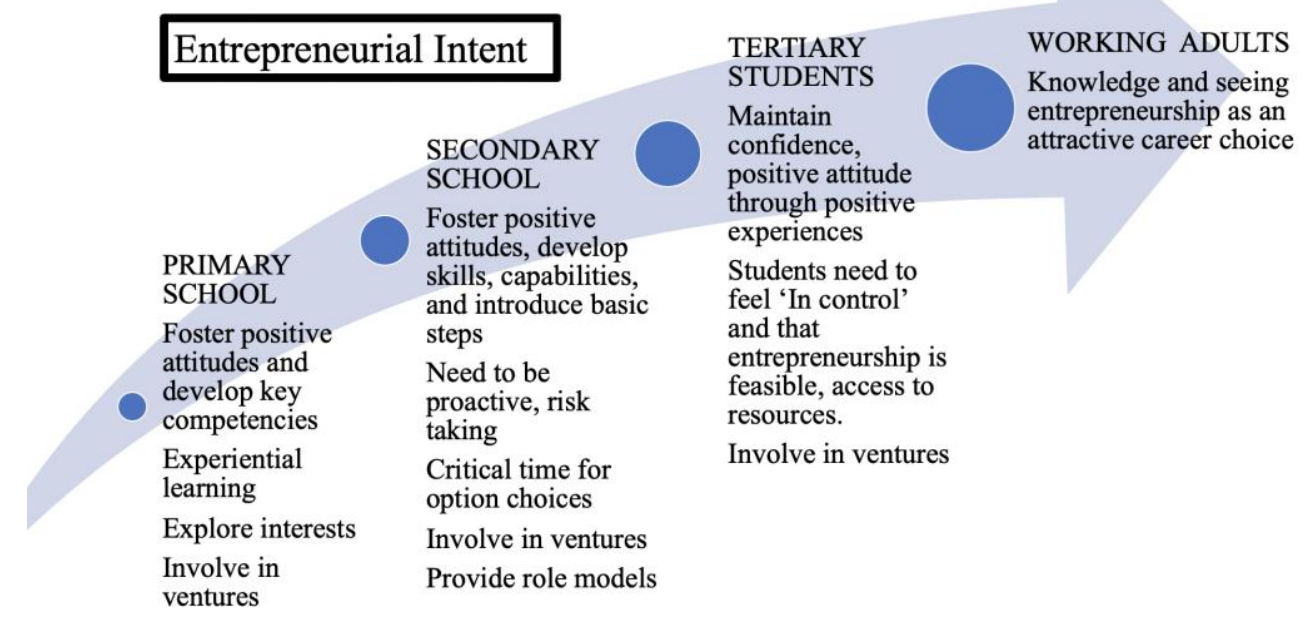

Figure 5. Recommendations for fostering entrepreneurial intent in eight international studies (Author based on work by Barba-Sánchez et al., 2016; Chuah et al., 2015; Ho et al., 2018; Huber et al., 2014; Lanero et al., 2011; Miralles et al., 2016; Mørch, 2018; Thompson \& Kwong, 2016) 
However, five studies found that entrepreneurship education could reduce intent as students felt it would be too hard to be an entrepreneur. While this was explained as a reality check by Huber et al. (2014), Nabi et al. (2018), and Oosterbeek et al. (2010), Nabi also highlights that in their study they found a single negative experience such as a tutor focusing too much on business failure could deter students. As Chuah et al. (2015) explains, their study showed intent can be negatively affected by entrepreneurship education because students need to be supported and encouraged with learning experiences that allow them to develop.

McNaughton and Yun (2018), found New Zealanders to have very low participation in entrepreneurship education at university (Table 4). When this was examined further in the Global University Entrepreneurial Spirit Survey report (Sieger et al., 2019), New Zealand students had the highest rate amongst countries in the study for not taking any entrepreneurship education courses and graduates future five year plan still had lower entrepreneurship rates globally, ranking 45th out of 54 countries (Table 4).

Table 4

Average Rate of Entrepreneurial Intent in New Zealand (McNaughton \& Yun, 2018) compared with the Global University Entrepreneurial Spirit Students' Survey (GUESSS) (Sieger et al., 2019).

\begin{tabular}{lcc}
\hline Findings & Guess & NZ (n=1920) \\
\hline Have not taken any Entrepreneurship education courses & $50.3 \%$ & $79.9 \%$ \\
Undergraduates who want to be employees & $79 \%$ taken from & $80 \%$ \\
Postgraduates who wish to be employees & GUESS report & $85.7 \%$ \\
Undergraduates with entrepreneurial intent & $9.3 \%$ & $3.9 \%$ \\
Postgraduates with entrepreneurial intent & $10.2 \%$ & $4.9 \%$ \\
\hline
\end{tabular}

Employment was seen as a more desirable option than entrepreneurship in two countries where the opposite would be expected. In Spain, entrepreneurship education initiatives were few and far between and employment was seen as the safest option, even though unemployment was as high as 55\% in under 25 year olds (Sánchez-García et al., 2013). Whereas, in Norway $90 \%$ of secondary schools provide entrepreneurship education (Johansen \& Schanke, 2013) and employment was preferred as the easiest option because jobs were plentiful (Støren, 2014).

\subsection{Pedagogical Approach to Entrepreneurship Education}

The pedagogy of teaching entrepreneurship is critical in terms of student engagement and motivation. A study in Singapore, compared 142 students 13-16 years of age who were trained in entrepreneurship by teachers and hired external enterprise education providers with 186 students who were not (Ho et al., 2018). Students' efficacy and alertness was found to be significantly higher with training (Figure 6).

Four studies recommended using a combination of for entrepreneurship and through entrepreneurship approaches and four studies recommended using just the through entrepreneurship approach (Figure 7).

In Sweden, definitions about, for and through were thought to be too focused on pedagogy and instead compared three foundational entrepreneurship approaches, value creation, venture creation and idea and artefact creation (Lackéus, 2020). Value creation was found to be the most effective tool in developing motivation, knowledge and skills in 1048 students 8-15 years of age. Students felt passionate about contributing to others and society. Venture creation was also valued by students as it connected to the realities of starting up a business. 


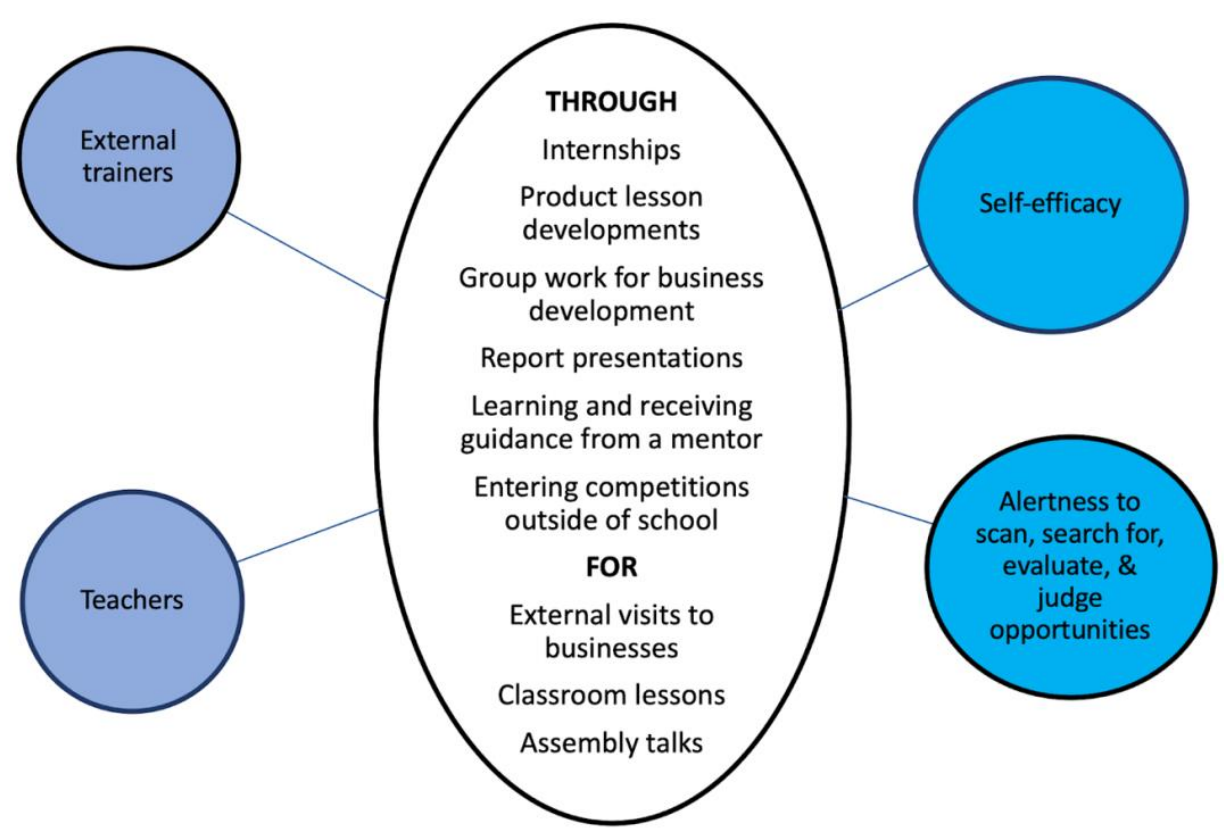

Figure 6. 'For' and 'through' approach to entrepreneurship education in Singapore with 13-16 year old students ( $\mathrm{n}=142)$ (Author based on work by Ho et al., 2018)

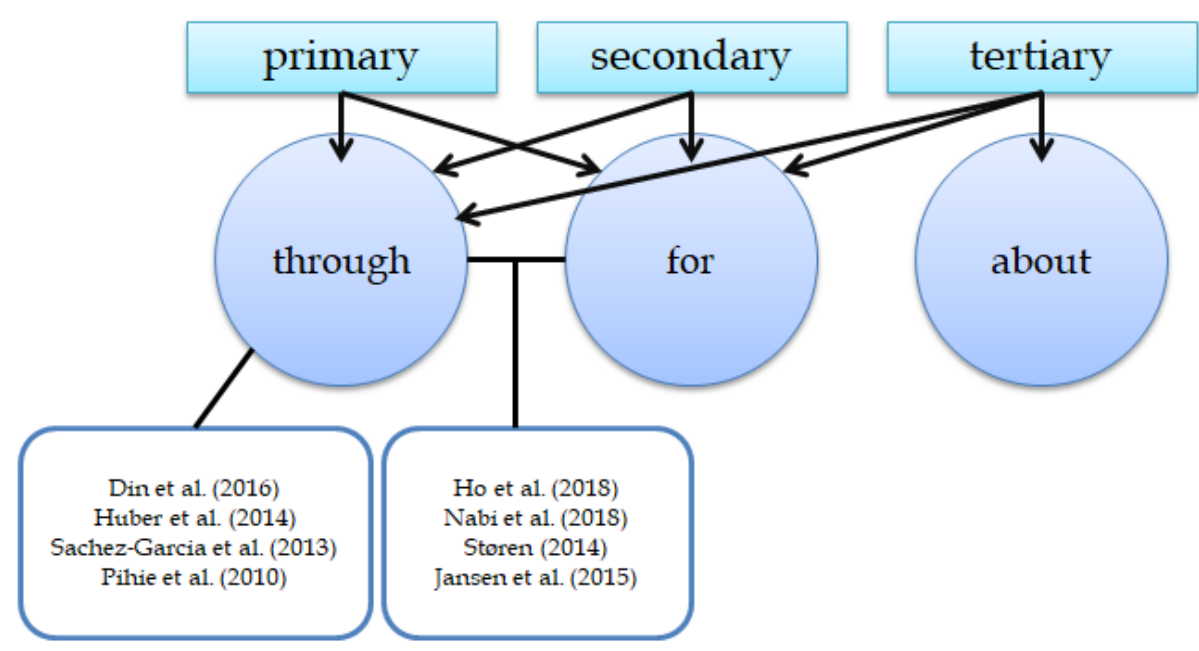

Figure 7. A comparison of entrepreneurship education approaches used in eight of the studies with arrows to indicate what has been recommended for education level

\subsection{Teacher Confidence, Knowledge and Capacity}

Teachers lack experience and confidence to develop effective entrepreneurship programmes. Issues in entrepreneurship education were described in 11 studies and these could be resolved through training teachers and principals, improving curricula, and fostering teacher commitment (Figure 8). 


\begin{tabular}{|c|c|}
\hline Issues in entrepreneurship pedagogy & Developing entrepreneurship education \\
\hline 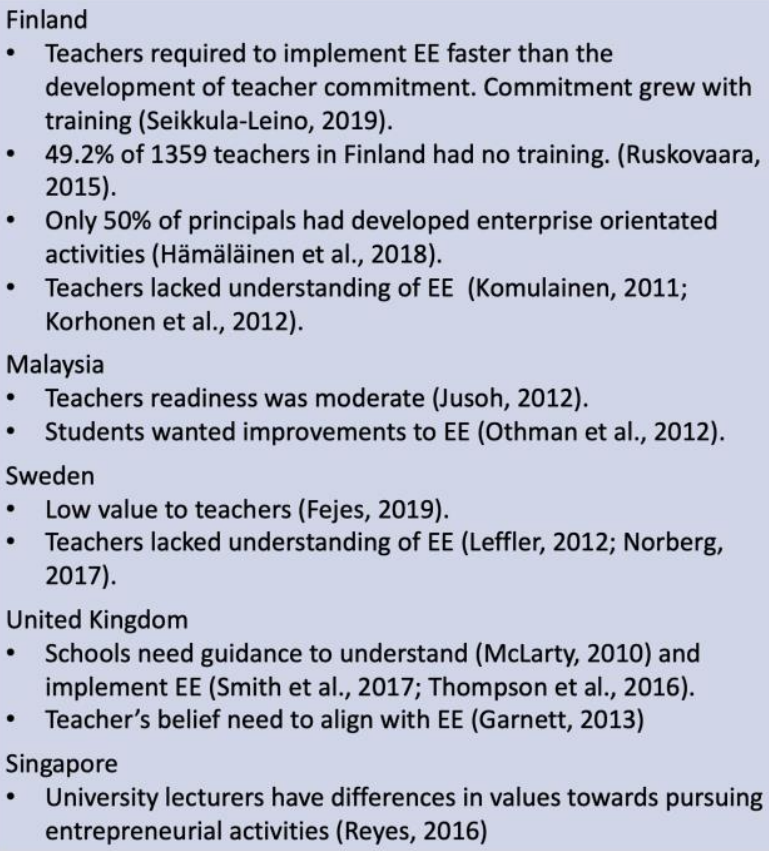 & $\begin{array}{l}\text { Finland } \\
\text { - Those who had trained were 3-4xs more advanced in } \\
\text { entrepreneurship education methods (Ruskovaara, 2013). } \\
\text { - Trained Principals increase and support EE (Minna et al., 2018). } \\
\text { Singapore } \\
\text { - Hired trainers to work alongside teachers (Ho et al., 2018). } \\
\text { - } 93 \% \text { university lecturers want university-enterprise co- } \\
\text { operation and } 85 \% \text { agree the relationship allows research } \\
\text { project size and scope to be enlarged. (Mok, 2015) } \\
\text { New Zealand } \\
\text { - Schools had clearly defined vision of EE. Teachers worked with } \\
\text { researchers which resulted in renewed energy and enthusiasm } \\
\text { (Kirkley, 2017). } \\
\text { Norway } \\
\text { - Teacher as mentor guided learning (Riese, 2013). } \\
\text { Value creation an effective tool to motivate students and } \\
\text { improve, knowledge and skills acquisition (Lackéus, 2020) } \\
\text { Spain } \\
\text { - Institutions act as an environmental support of EE (Lanero et } \\
\text { al., 2011). } \\
\text { Malaysia } \\
\text { - EE requires a shift in pedagogy (Ahmad, 2013). }\end{array}$ \\
\hline
\end{tabular}

Figure 8. Issues in entrepreneurship education (EE) pedagogy with developing EE, including training, curricula and commitment

\section{Discussion and Conclusion}

Entrepreneurship education has the potential to develop students' competencies, knowledge and skills to confidently act on opportunities, address issues and solve problems that have arisen in their communities. However, this literature review found barriers in developing students' entrepreneurship capabilities and intent, and in supporting teachers to implement effective programmes.

There is no knowing how long or how deep the impact of the current economic depression caused by the COVID-19 pandemic will penetrate our globe and standard of living (Baldwin \& di Mauro, 2020). Entrepreneurship education in public schooling and universities requires urgent attention and focus to support and enable young people to understand how they can adapt to a changing environment, (Rieckmann, 2020) to lead and understand the emerging possibilities (Scharmer et al., 2020). This literature review found benefits for students who experience entrepreneurship education including, the development of self-efficacy, motivation and engagement, positive attitudes for identifying and acting on opportunities with knowledge, skills and creativity. Future success in the aftermath of a pandemic requires students to be adaptable, resilient (OECD, 2020), opportunistic, innovative and entrepreneurial which are all capabilities that can be developed through effective entrepreneurship education (Lackéus, 2020; Maritz et al., 2020).

The current literature reveals clear evidence that in order for entrepreneurship education to be effective student's need positive 'hands on' experiences to be fostered throughout schooling and into university so that they can build entrepreneurial intent and confidence. Entrepreneurship education helps to foster the intent of students to be entrepreneurs (Marire, 2015; Rauch \& Hulsink, 2015) and can take time to form (Gorgievski \& Stephan, 2016). However, this review of the literature found that university graduates have been emerging with high rates of intent to be employees and not business developers or owners (McNaughton \& Yun, 2018; Sieger et al., 2019). The OECD (2019) 'Employment Outlook 2019' report repeatedly states that young people are more at risk than other age groups of being underpaid and underemployed, especially if they lack education. While Morgan (2020) argues that students need knowledge plus socialism and not entrepreneurship for future success, Lackéus (2017) explains entrepreneurship can be developed 
for the purpose of serving others, develops skills and competencies and enhances student engagement. The multitude of issues that have arisen for families in this current global crisis requires teachers to be receptive to learners (Bryant et al., 2020; UNESCO, 2020) who when placed at the centre of learning can connect to being entrepreneurial for their communities (Mika et al., 2017) and for employment in a post-Covid world.

This study found that teachers need opportunities to build confidence, knowledge and capacity in order to develop effective entrepreneurship education learning experiences that are relevant to today's students and prepares them for future life challenges. . Teachers may not have experienced any form of entrepreneurship activity themselves and therefore lack the confidence to facilitate this learning as they may have stronger content and pedagogical knowledge in traditional curriculum areas. Entrepreneurship education can be effectively implemented with experiential approaches to learning, such as value creation (Lackéus, 2020) and support from external trainers to develop programmes (Ho et al., 2018). Further studies are needed to understand the attitudes and values of teachers who are effectively supporting students to engage in entrepreneurship education. Developing research knowledge and expertise of the specific resources and training that is required to encourage teachers who have not yet gained experience would provide value for policy makers and school leaders to encourage this cross curricular approach to learning. A deeper understanding and models of initiatives that encourage young people to develop confidence in entrepreneurial endeavours in a rapidly changing and uncertain economic environment is urgently needed and an area that would be prudent for education leaders to focus. Further study into current and potential influences that foster entrepreneurship intention in the young people of today, such as online platforms, may help to connect their interests to the classroom.

Acknowledgement. This article is the extended version of a paper presented at the International Congress of Pedagogical Research ICOPR'2020 held at Duzce University in June, 2020.

\section{References}

Ajzen, I. (1991). The theory of planned behavior. Organizational behavior and human decision processes, 50(2), 179-211. https://doi.org/10.1016/0749-5978(91)90020-T

Akkermans, J., Richardson, J., \& Kraimer, M. (2020). The Covid-19 crisis as a career shock: Implications for careers and vocational behavior. Journal of Vocational Behavior, 119(1), 1-5, Article 103434. https://doi.org/10.1016/j.jvb.2020.103434

Baker, S. R., Bloom, N., Davis, S. J., \& Terry, S. J. (2020). Covid-induced economic uncertainty (Working Paper No. 26983). (Economic Fluctuations and Growth, Issue April 2020). http://www.nber.org/papers/w26983

Baldwin, R., \& di Mauro, B. W. (2020). Introduction. In R. Baldwin \& B. W. di Mauro (Eds.), Economics in the Time of COVID-19 (pp. 1-30). VoxEU CEPR Press.

Barba-Sánchez, V., Atienza-Sahuquillo, C., McCracken, M., \& Matlay, H. (2016). The development of entrepreneurship at school: the Spanish experience. Education+ Training, 5(7/8), 783-796. https:/ / doi.org/10.1108/ET-01-2016-0021

Bhattacharjee, A., \& Jahanshah, A. (2020, April 3). The COVID-19 crisis brings spring season for translucent activity. Does it result exogenous uncertainty for the entrepreneurs and bound-less commodity pricing! Social Science Research Network. https:/ / doi.org/10.2139/ssrn.3568972

Braun, V., \& Clarke, V. (2006). Using thematic analysis in psychology. Qualitative research in psychology, 3(2), 77-101. https:/ / doi.org/10.1191/1478088706qp063oa

Bryant, J., Chen, L., Dorn, E., \& Hall, S. (2020, April 21). Now that the immediate rush of closing K-12 schools is over, school systems must focus on four major priorities - and put equity front and centre. McKinsey $\mathcal{E}$ Company. https://www.mckinsey.com/industries/public-sector/our-insights/school-system-prioritiesin-the-age-of-coronavirus

Caird, S. (1990). What does it mean to be enterprising. British Journal of Management, 1(1), 137-145. https://doi.org/10.1111/j.1467-8551.1990.tb00002.x 
Chuah, C. W., Ting, H., Alsree, S. R., \& Cheah, J. H. (2015, December 22). Factors affecting entrepreneurial intention of Malaysian university student. Paper presented at Conference on Business Management Research II (CBMR II 2015), Kedah, Malaysia.

Dahlstedt, M., \& Hertzberg, F. (2012). Schooling entrepreneurs: Entrepreneurship, governmentality and education policy in Sweden at the turn of the millennium. Journal of Pedagogy, 3(2), 242-262. https://doi.org/10.2478/v10159-012-0012-x

Dai, R., Hu, J., \& Zhang, X. (2020). The Impact of Coronavirus on China's SMEs: Findings from the Enterprise. Center for Global Development. https://www.cgdev.org/sites/default/files/impact-coronaviruschinas-smes-findings-from-esiec.pdf

Davidsen, H. M. (2015). Entrepreneurship and Learning [Conceptual paper]. https://www.ucviden.dk/portal/en/publications/entrepreneurship-and-learning(1a40d15a-0e7e-482caf7f-31d910684611).html

Din, B. H., Anuar, A. R., \& Usman, M. (2016). The effectiveness of the entrepreneurship education program in upgrading entrepreneurial skills among public university students. Procedia-Social and Behavioral Sciences, 224(1), 117-123. https:// doi.org/10.1016/j.sbspro.2016.05.413

Elahi, T. (2019). Critically evaluating the approach of teaching $\mathcal{E}$ developing successful entrepreneurs https://doi.org/10.13140/RG.2.2.15869.72161

Fejes, A., Nylund, M., \& Wallin, J. (2019). How do teachers interpret and transform entrepreneurship education? Journal of Curriculum Studies, 51(4), 554-566. https://doi.org/10.1080/00220272.2018.1488998

Fernandes, N. (2020, April, 13). Economic effects of coronavirus outbreak (COVID-19) on the world economy. 2020. Available at SSRN. https://doi.org/10.2139/ssrn.3557504

Gibb, A., \& Ramsey, E. (2011). Concepts into practice: meeting the challenge of development of entrepreneurship educators around an innovative paradigm. International Journal of Entrepreneurial Behavior \& Research, 17(2), 146-165. https:/ / doi.org/10.1108/13552551111114914

Gorgievski, M. J., \& Stephan, U. (2016). Advancing the psychology of entrepreneurship: A review of the psychological literature and an introduction. Applied Psychology, 65(3), 437-468. https://doi.org/10.1111/apps.12073

Gössling, S., Scott, D., \& Hall, C. M. (2020). Pandemics, tourism and global change: a rapid assessment of COVID-19. Journal of Sustainable Tourism, Advance online publication, 1-20. https://doi.org/10.1080/09669582.2020.1758708

Haeffele, S., Hobson, A., \& Storr, V. H. (2020, April 28). Coming Back from COVID-19: Lessons in Entrepreneurship from Disaster Recovery Research. Mercatus Center Research Paper Series, Mercatus Special Edition Policy Brief, 1-7. https:// doi.org/10.2139/ssrn.3592966

Hämäläinen, M., Ruskovaara, E., \& Pihkala, T. (2018). Principals Promoting Entrepreneurship Education: The Relationships between Development Activities and School Practises. Journal of Entrepreneurship Education, https:/ / scholar.google.co.nz/scholar?start=40\&q=Finland+schools+entrepreneurship\&hl=en\&as_sdt=0,5

Hannon, P. D. (2005). Philosophies of enterprise and entrepreneurship education and challenges for higher education in the UK. The International Journal of Entrepreneurship and Innovation, 6(2), 105-114.

Harrington, C., \& Maysami, R. (2015). Entrepreneurship education and the role of the regional university. Journal of Entrepreneurship Education, 18(2). https://www.abacademies.org/articles/jeevol1822015.pdf

Ho, M. H., Uy, M. A., Kang, B. N. Y., \& Chan, K. Y. (2018). Impact of entrepreneurship training on entrepreneurial efficacy and alertness among adolescent youth. Frontiers in Education, 3(13), 1-10. https://doi.org/10.3389/feduc.2018.00013

Ho, Y. P., Low, P. C., \& Wong, P. K. (2014). Do University Entrepreneurship Programs Influence Students' Entrepreneurial Behavior? An Empirical Analysis of University Students in Singapore. In S. Hoskinson \& D. F. Kuratko (Eds.), Innovative Pathways for University Entrepreneurship in the 21st Century (Vol. 24, pp. 6587). Emerald Group Publishing Limited. https:// doi.org/10.1108/S1048-473620140000024003

Huber, L. R., Sloof, R., \& Van Praag, M. (2014). The effect of early entrepreneurship education: Evidence from a field experiment. European Economic Review, 72(1), 76-97. https://doi.org/10.1016/j.euroecorev.2014.09.002

Ierapetritis, D. G. (2017). Entrepreneurship education at school: a case study on secondary education in Greece. World Review of Entrepreneurship, Management and Sustainable Development, 13(2-3), 271-289. https://doi.org/10.1504/WREMSD.2017.083023

Jansen, S., Van De Zande, T., Brinkkemper, S., Stam, E., \& Varma, V. (2015). How education, stimulation, and incubation encourage student entrepreneurship: Observations from MIT, IIIT, and Utrecht University. 
The International Journal of Management Education, 13(2), 170-181. https://doi.org/10.1016/j.ijme.2015.03.001

Johansen, V., \& Schanke, T. (2013). Entrepreneurship education in secondary education and training. Scandinavian Journal of Educational Research, 57(4), 357-368. https://doi.org/10.1080/00313831.2012.656280

Jones, B., \& Iredale, N. (2014). Enterprise and entrepreneurship education: towards a comparative analysis. Journal of Enterprising Communities: People and Places in the Global Economy, 8(1), 34-50. https://doi.org/10.1108/jec-08-2012-0042

Jusoh, R. (2012). Effects of Teachers' Readiness in Teaching and Learning of Entrepreneurship Education in Primary Schools. International Interdisciplinary Journal of Education, 1(7), 98-102. https:// doi.org/10.12816/0002885

Kirkley, W. W. (2017). Cultivating entrepreneurial behaviour: entrepreneurship education in secondary schools. Asia Pacific Journal of Innovation and Entrepreneurship, 11(1), 17-37. https://doi.org/10.1108/apjie04-2017-018

Kleiman. (2015). Excellence in diversity: A report celebrating the diversity of UK higher education. https://www.guildhe.ac.uk/wp-content/uploads/2015/07/Excellence-in-diversity-ExecutiveSummary-2-July-2015.pdf

Komulainen, K., Korhonen, M., \& Räty, H. (2009). Risk-taking abilities for everyone? Finnish entrepreneurship education and the enterprising selves imagined by pupils. Gender and Education, 21(6), 631-649. https://doi.org/10.1080/09540250802680032

Lackéus, M. (2015). Entrepreneurship in education: What, why, when, how. https://www.oecd.org/cfe/leed/BGP_Entrepreneurship-in-Education.pdf

Lackéus, M. (2017). Does entrepreneurial education trigger more or less neoliberalism in education? Education+ Training, 59(6). https://doi.org/10.1108/ET-09-2016-0151

Lackéus, M. (2020). Comparing the impact of three different experiential approaches to entrepreneurship in education. International Journal of Entrepreneurial Behavior \& Research, Advance online publication. https:// doi.org/10.1108/IJEBR-04-2018-0236

Lackéus, M., \& Middleton, K. W. (2015). Venture creation programs: bridging entrepreneurship education and technology transfer. Education+ Training, 57(1), 48-73. https://doi.org/10.1108/ET-02-2013-0013

Lanero, A., Vázquez, J. L., Gutiérrez, P., \& García, M. P. (2011). The impact of entrepreneurship education in European universities: an intention-based approach analyzed in the Spanish area. International Review on Public and Nonprofit Marketing, 8(2), 111-130. https:/ / doi.org/10.1007/s12208-011-0067-8

Lee, K., Hebaishi, G., \& Hope, J. (2015). The role of senior management in developing and achieving a successful enterprise education programme? Education+ Training, 57(7), 791-811. https://doi.org/10.1108/ET-11-2014-0139

Marire, E. (2015). A Comparison of Entrepreneurial Intentions of Generation Y Students in South Africa and Zimbabwe North-West University, Vaal Triangle Campus]. South Africa.

Maritz, A., Perenyi, A., de Waal, G., \& Buck, C. (2020). Entrepreneurship as the Unsung Hero during the Current COVID-19 Economic Crisis: Australian Perspectives. Sustainability, 12(11), 1-9. https://doi.org/10.3390/su12114612

McKee, M., \& Stuckler, D. (2020). If the world fails to protect the economy, COVID-19 will damage health not just now but also in the future. Nature Medicine, 26(1), 640-648. https://doi.org/10.1038/s41591-0200863-y

McNaughton, R., \& Yun, A. (2018). Student entrepreneurship at the University of Auckland 2018: Global University Entrepreneurial Spirit Students' Survey 2018-National Report New Zealand. http://www.guesssurvey.org/resources/nat_2018/GUESSS_Report_2018_NewZealand.pdf

Mika, J. P., Warren, L., Foley, D., \& Palmer, F. R. (2017). Perspectives on indigenous entrepreneurship, innovation and enterprise. Journal of Management $\mathcal{E}$ Organization, 23(6), 767-773. https://doi.org/10.1017/jmo.2018.4

Ministry of Education. (2011, November). The New Zealand curriculum update 15: The future focus principle. The Education Gazette: Tukutuku Körero, (15). https://nzcurriculum.tki.org.nz/Curriculumresources/NZC-Updates

Miralles, F., Giones, F., \& Riverola, C. (2016). Evaluating the impact of prior experience in entrepreneurial intention. International Entrepreneurship and Management Journal, 12(3), 791-813. https://doi.org/10.1007/s11365-015-0365-4 
Moberg, K. (2014). Two approaches to entrepreneurship education: The different effects of education for and through entrepreneurship at the lower secondary level. The International Journal of Management Education, 12(3), 512-528. https:// doi.org/10.1016/j.ijme.2014.05.002

Mok, K. H. (2015). The quest for global competitiveness: promotion of innovation and entrepreneurial universities in Singapore. Higher Education Policy, 28(1), 91-106. https://doi.org/10.1057/hep.2014.30

Mørch, J. (2018). Entrepreneurship education beyond university campuses: examining the results of an independent entrepreneurship education programme-and its implications for how we educate the future entrepreneurs we need [Masters, Norwegian School of Economics]. Norway.

Morgan, J. (2020, June 20). We don't need no disruptive education. Schooling capitalism: exploring new educational cultures. https://schoolingcapitalism.wordpress.com/2020/06/20/we-dont-need-nodisruptive-education/

Nabi, G., Walmsley, A., Liñán, F., Akhtar, I., \& Neame, C. (2018). Does entrepreneurship education in the first year of higher education develop entrepreneurial intentions? The role of learning and inspiration. Studies in Higher Education, 43(3), 452-467. https:/ / doi.org/10.1080/03075079.2016.1177716

Nseobot, I. R., Simeon, I. I., Effiong, A. I., Frank, E. I., Ukpong, E. S., \& Essien, M. O. (2020). COVID-19: The Aftermath for Businesses in Developing Countries. International Journal of Business Education and Management Studies, 5(1), 43-49. https:// ssrn.com/abstract=3592603

O'Leary, S. (2012, April 12 - 13). Using entrepreneurship to enhance the employability of scientists and engineers 1st Annual Conference on th Aiming for Excellence in STEM Learning and Teaching, London.

OECD. (2019). OECD Employment Outlook 2019. OECD Publishing. https://doi.org/10.1787/9ee00155-en

OECD. (2020). Coronavirus special edition: Back to school. Trends Shaping Education Spotlight 21. https://www.oecd-ilibrary.org/content/paper/339780fd-en

Oosterbeek, H., Van Praag, M., \& Ijsselstein, A. (2010). The impact of entrepreneurship education on entrepreneurship skills and motivation. European Economic Review, 54(3), 442-454. https:// doi.org/10.1016/j.euroecorev.2009.08.002

Osiri, J., McCarty, M. M., Davis, J., \& Osiri, J. E. (2015). Entrepreneurship mix and classifying emerging sub$\begin{array}{lllll}\text { fields. } & \text { Academy of Journal, } & \text { 21(1), } & \text { 1-12. }\end{array}$ https://www.abacademies.org/articles/aejvol21no12015.pdf\#page=7

Othman, N., Hashim, N., \& Ab Wahid, H. (2012). Readiness towards entrepreneurship education. Education+ Training, 54(8/9), 697-708. https://doi.org/10.1108/00400911211274837

Pepin, M. (2018). Learning to be enterprising in school through an inquiry-based pedagogy. Industry and Higher Education, 32(6), 418-429. https://doi.org/10.1177/0950422218802536

Pihie, Z. L., \& Bagheri, A. (2011). Malay secondary school students' entrepreneurial attitude orientation and entrepreneurial self-efficacy: A descriptive study. Journal of Applied Sciences, 11(2), 316-322. https:// doi.org/10.3923/jas.2011.316.322

Piperopoulos, P., \& Dimov, D. (2015). Burst bubbles or build steam? Entrepreneurship education, entrepreneurial self-efficacy, and entrepreneurial intentions. Journal of Small Business Management, 53(4), 970-985.

Rauch, A., \& Hulsink, W. (2015). Putting entrepreneurship education where the intention to act lies: An investigation into the impact of entrepreneurship education on entrepreneurial behavior. Academy of management learning \& education, 14(2), 187-204. https:// doi.org/10.5465/amle.2012.0293

Reimers, F. M., \& Schleicher, A. (2020). A framework to guide an education response to the COVID-19 Pandemic of $2020 . \quad$ OECD Publishing. Retrieved from https://www.hm.ee/sites/default/files/framework_guide_v1_002_harward.pdf

Reyes, C. N. (2016). Framing the entrepreneurial university: the case of the National University of Singapore. Journal of Entrepreneurship in Emerging Economies, 8(2), 134-161. https://doi.org/10.1108/JEEE-09-20150046

Rieckmann, M. (2020, May 7, 2020). Innovative Learning Approaches to foster sustainable entrepreneurship competencies with regard to the SDGs in Higher Education [Webinar]. Intrinsic project. https://vimeo.com/387802522

Riese, H. (2013). Mini-enterprise projects: friendship, business and learning. Journal of Education and Work, 26(4), 453-471. https:/ / doi.org/10.1080/13639080.2011.653557

Robertson, G. (2020). Grant Robertson's 2020 Budget Speech. Labour. Retrieved from https://www.labour.org.nz/budget2020-grant-robertson-

speech?utm_campaign=200514_budgetspeeches\&utm_medium=email\&utm_source=nzlabour 
Sánchez-García, J. C., Arnedo, R. A., \& Hernández-Sánchez, B. (2013). Activities and programs of entrepreneurship education in Spain. American Journal of Entrepreneurship, 6(2), 73.

Scharmer, O., Stark, W., \& Sailer, K. (2020, May 7). Transformational Learning and the Future of Entrepreneurial HEIs [Webinar]. HEInnovate.

Sieger, P., Fueglistaller, U., Zellweger, T., \& Braun, I. (2019). Global Student Entrepreneurship 2018: Insights from 54 Countries (Global GUESSS Report, Issue. Retrieved from http://www.guesssurvey.org/publications/publications/international-reports.html

Sirelkhatim, F., \& Gangi, Y. (2015). Entrepreneurship education: A systematic literature review of curricula contents and teaching methods. Cogent Business \& Management, 2(1), 1-11, Article 1052034. https:/ / doi.org/10.1080/23311975.2015.1052034

Smith, S., \& Price, A. (2011). The Institute for Enterprise Centre for Excellence in Teaching and Learning: history, context, work and issues for future sustainability. In R. Kill \& K. O'Rourke (Eds.), Inspiring Enterprise: Transforming Enterprise Education at Leeds Metropolitan University (pp. 2-18). Leeds Met Press.

Støren, L. A. (2014). Entrepreneurship in higher education-impacts on graduates' entrepreneurial intentions, activity and learning outcome. Education + Training, 56(8/9). https://doi.org/10.1108/ET-06-2014-0070

Thompson, P., \& Kwong, C. (2016). Compulsory school-based enterprise education as a gateway to an entrepreneurial career. International Small Business Journal, 34(6), 838-869. https://doi.org/10.1177/0266242615592186

Tranfield, D., Denyer, D., \& Smart, P. (2003). Towards a methodology for developing evidence-informed management knowledge by means of systematic review. British Journal of Management, 14(3), 207-222. https://doi.org/10.1111/1467-8551.00375

UNESCO. (2020). COVID-19 education response: Preparing the reopening of schools: resource paper. https://unesdoc.unesco.org/ark:/48223/pf0000373401

Van Lancker, W., \& Parolin, Z. (2020). COVID-19, school closures, and child poverty: a social crisis in the making. The Lancet Public Health, 5(5), e243-e244. https:// doi.org/10.1016/S2468-2667(20)30084-0

Wade, A. (2020, May 25). New Zealand joins 'early movers intiative' to discuss Covid-19 response with other successful countries. Retrieved from https:/ / www.nzherald.co.nz/nz/news/article.cfm?c_id=1\&objectid=12334686

$\begin{array}{lllll}\text { Worldometer. (2020). Covid-19 Coronavirus pandemic. } & \text { Retrieved from }\end{array}$ https://www.worldometers.info/coronavirus/

$\mathrm{Wu}$, Y.-C. J., \& Wu, T. (2017). A decade of entrepreneurship education in the Asia Pacific for future directions in theory and practice. Management Decision, 55(7). https://doi.org/10.1108/MD-05-2017-0518

Yu, M.-C., Goh, M., Kao, H.-Y., \& Wu, W.-H. (2017). A comparative study of entrepreneurship education between Singapore and Taiwan. Management Decision, 55(7). https://doi.org/10.1108/MD-06-2016-0415

Zhou, L., Wu, S., Zhou, M., \& Li, F. (2020). 'School's Out, But Class' On', The Largest Online Education in the World Today: Taking China's Practical Exploration During The COVID-19 Epidemic Prevention and Control As an Example. Best Evid Chin Edu 2020, 4(2), 501-519. https://doi.org/10.2139/ssrn.3555520 